\title{
A Content-Analysis Approach for Exploring Usability Problems in a Collaborative Virtual Environment
}

Dalma Geszten ${ }^{1}$, Anita Komlódi ${ }^{2}$, Károly Hercegfi ${ }^{1}$, Balázs
Hámornik $^{1}$, Alyson Young
, Máté Köles ${ }^{1}$, Wayne G Lutters ${ }^{2}$

${ }^{1}$ Department of Ergonomics and Psychology, Budapest University of Technology and Economics

Magyar tudósok körútja 2, H-1117 Budapest, Hungary

gesztend@erg.bme.hu, hercegfi@erg.bme.hu, hamornik@erg.bme.hu, kolesm@erg.bme.hu

${ }^{2}$ Department of Information Systems, University of Maryland

Baltimore County (UMBC), 1000 Hilltop Cir, Baltimore, MD 21250, USA

komlodi@umbc.edu, lutters@umbc.edu

${ }^{3}$ Department of Human-Centered Computing, Indiana University - Purdue

University Indianapolis (IUPUI)

420 University Blvd, Indianapolis, IN 46202, USA

youngaly@iupui.edu

Abstract: As Virtual Reality (VR) products are becoming more widely available in the consumer market, improving the usability of these devices and environments is crucial. In this paper, we are going to introduce a framework for the usability evaluation of collaborative $3 D$ virtual environments based on a large-scale usability study of a mixedmodality collaborative VR system. We first review previous literature about important usability issues related to collaborative $3 D$ virtual environments, supplemented with our research in which we conducted 122 interviews after participants solved a collaborative virtual reality task. Then, building on the literature review and our results, we extend previous usability frameworks. We identified twelve different usability problems, and based on the causes of the problems, we grouped them into three main categories: VR environment-, device interaction-, and task-specific problems. The framework can be used to guide the usability evaluation of collaborative VR environments.

Keywords: virtual reality; usability evaluation; content-analysis; CAVE 


\section{Introduction}

Virtual reality (VR) research has several decades of history, although interest in this area varied over time. The Gartner hype cycle for emerging technologies shows the process a new technology has to go through to become widely popular [22]. These new information technologies have reached a higher level of complexity which requires introducing novel approaches, such as cognitive infocommunications (CogInfoCom) [1]. In the terms of the Gartner hype cycle VR is now in the "Slope of enlightenment" phase. This phase signifies that a particular technology has successfully gone through the "Trough of disillusionment" phase and now it is becoming more and more clear in what aspects of life virtual reality is useful. The real life usage of virtual reality environments and devices is more and more widespread [2] [3] [5] [6] [8] [16] [17]. As virtual reality products are becoming available for the consumer market and the user base is expanding, it is especially important that these devices are easy to learn and natural to use. What level of cognitive compatibility is reached between the user and the system, how users interact with the VR environment, what their difficulties are in different situations and how these problems can be solved are enormous challenges. For these reasons, it is essential to examine the usability of virtual reality environments.

Bowman defines usability in virtual reality as "the characteristics of an artifact (usually a device, interaction, technique or complete UI - User Interface) that affect the user's use of that artifact" [4]. Providing a set of usability goals helps both designers and evaluators of technology to guide the interaction design of these tools. The goal of this paper is to provide a framework of usability goals for Collaborative Virtual Environments (CVEs).

We will first review related research examining usability studies and goals of VR technologies, and then present an expanded usability framework for CVEs based on a usability study of one particular CVE.

\section{Related Research}

While virtual reality has been well studied, the characteristics of collaboration in these environments and the ways in which usability can enhance it is a relatively new research area. One reason for this may be the immaturity of the technology; collaborative virtual environments (CVEs) are relatively young, which means they suffer from usability issues [26]. Despite this there have been a number of attempts to define usability problems within these environments. Next, we provide an overview of these. 


\subsection{Usability Problems in Collaborative Virtual Environments}

From the overview of the literature we can see that the most serious usability issues of collaborative virtual environments are: 1) navigation and manipulation problems, 2) technical problems (both UI and input devices), 3) visual awareness and visibility problems, and 4) learnability problems.

\subsubsection{Navigation and Manipulation}

Navigation and manipulation are the most important activities users have to carry out in a virtual space. Navigation allows users to move around and manipulation makes interaction with virtual objects possible. Without these two functions users are not able to interact with the spaces. Several usability problems occurred related to navigation and manipulation in different collaborative virtual environments. Participants found both moving around in the environment and moving objects in the environment difficult. Spatial navigation problems were reported in a collaborative virtual environment both in immersive spaces [32] and in desktop-based interaction with virtual environments [12] [33] [31]. Typical problems included the inability to reach a desired location [32], becoming disoriented [12], and inability to move or aimlessly moving about the space [33]. Manipulation problems also occurred in both immersive [32], desktop and HMD settings [11]. Grabbing and moving objects was often not well supported and difficult for participants. Navigation and manipulation problems are complex because they can be caused by several factors like input devices, the environment itself (HMD, CAVE or desktop interface), learnability and personality factors. As these functions are crucial to the use of virtual environments, they are important to mitigate.

\subsubsection{Technical Problems Impacting Usability}

The immaturity of VR systems often leads to serious technical problems, which make collaboration impossible. Less serious problems can cause breakdowns in verbal communication when participants cannot hear each other which makes collaboration impossible [32] [21]. Another important area of technical problems is that of lags and delays, which can also make communication and collaboration difficult [32] Input devices also often caused technical problems, in some cases they were unreliable and stopped working on occasion, in other cases they were bulky and heavy and difficult to use [12]. The frequency of technical problems confirms Schroeder's statement [26] that collaborative virtual reality technology was not yet mature enough at the time of these studies to prevent serious technical problems. While it is expected that technical problems will lead to usability issues, it is important to highlight how the immaturity of CVEs present ongoing difficulties for users. 


\subsubsection{Visual Awareness and Visibility Problems}

Visual awareness and visibility problems also made collaboration and communication difficult. Visual awareness constitutes the ability to see collaborators, including facial expressions, gestures, and movements. When these are not visible to collaborators, communication and collaboration can break down [31]. Similarly Tromp et al. [32] reported that because the lack of facial expressions and body language, participants were sometimes confused about whose turn it was to talk. Phatic communication (beginning and ending of conversation) was really hard for participants in this environment. Better visual awareness and high quality audio should help alleviate these problems. When gestures cannot be seen from the partner's point of view, participants replaced the gestures with verbal communication [13]. Tromp also stated that participants supplemented physical actions with verbal descriptions because working together on a distant object was difficult [32]. In addition to the visibility of gestures, other visibility problems occurred: in some cases, the poor visibility of objects caused problems [13]. In another case, avatars obscured the partner's view of the environment [32]. Visibility problems are also linked to navigation. If the user can easily move around the space, they can try different views of an object. However, if navigation is limited visibility problems can become serious as well.

\subsubsection{Learnability Problems}

Learning how to navigate and manage the virtual environment is not easy. In Heldal's [12] and also in Tromp's study [32] participants reported that it was difficult and confusing for users to learn how to control the system with a keyboard and a mouse. In Heldal's study [12] participants did not manage to learn to avoid using the non-tracked hand to point or for other actions. In general, mapping navigation and manipulation functions to various devices usually not designed for immersive spaces makes learning the interaction methods difficult. There are contradictory results about how usability affects collaboration. Sometimes, despite serious usability issues, participants managed to collaborate effectively and reported that, although they noticed the problems they did not care about them [32]. In other cases collaboration was seriously affected by the usability problems of the virtual environments [31]. Fixing usability issues should still be a priority even though other factors seem to influence the success of the interaction.

\subsection{Presence and Copresence as Usability Factors in Virtual Environments}

Presence is the participant's sense of being there in the virtual environment [27] and copresence is the feeling of being there together [25] [26], a sense of others in the same place. Different theories consider different aspects of presence and 
copresence important. In his article, Schroeder [26] emphasizes that presence is a sensory experience, mainly auditory and visual and sometimes haptic. Sas and O'hare [24] argue that presence is determined by technological factors ("visual display characteristics such as image quality, image size, viewing distance, visual angle, motion, color, dimensionality, camera techniques; aural presentation characteristics like frequency range, dynamic range, signal-to-noise ratio, highquality audio, and dimensionality such as 3D sound") and human factors (empathy, absorption, creative imagination, willingness to experience presence). It is also important to note that a key factor of copresence is mutual awareness of others and their actions according to Kohonen-Aho [15]. Thus, presence and copresence are experienced by the user based on various features of the virtual environment and mediated by the user's characteristics. The common factor in these definitions is that they all highlight the importance of the sensory realism of the virtual environment. Presence is factor of the quality/reality of a virtual reality environment [29]. The design solutions which aim is to enhance the feeling of presence and copresence of participants in virtual environments mainly include designing as real-like environments as possible and providing sensory realism.

Bowman [4] and Heeter [9] state that presence should be considered a usability factor when designing or evaluating a virtual reality environment. Bowman [4] argues that when evaluating the usability of 3D user interfaces, measuring phenomena like presence and cybersickness is important because these are also part of the user experience of the interface. In their article, Shim \& Kim [28] go even further and suggest the idea of presence-driven VR development research, which aims to maximize presence in a given VR environment by manipulating different presence factors (like field of view and simulation level of detail) in a cost-effective way. Heeter [9] also suggests that developers and researchers should keep presence in mind when designing a virtual environment with thinking through several questions concerning the goals of presence, such disturbing factors in the environment which weaken or destroy the presence feeling of the participant.

The degree to which the user feels present in the environment marks the success of the environment. Usability problems will decrease the feeling of being present. If the user has to struggle with using the environment, s/he will not be able to experience the "reality" of the artificial environment. Thus, usability problems have a crucial role in the success of an immersive VR environment. Moreover, we also believe that in a collaborative setting the question of copresence is just as important as presence, which makes the problem even more complex. Thus, presence and copresence should be considered goals when designing VR environments and should be examined when evaluating the usability of these environments. 


\subsection{Stanney's Framework to Categorize Human Factors in VR}

Stanney [30] summarizes the most important human factors, that influence VR environment usage and performance in VR. These five factors contain: task characteristics, user characteristics, multi-modal interaction, health and safety issues, and the possible importance of new design metaphors. First, according to Stanney, the characteristics of the VR task determines the participant's performance. Some tasks are more suitable for a VR environment for example tasks that are related to information integration. Stanney states that the task characteristics is a key factor because it directly influences performance. Second, Stanney argues that there are three main user characteristics that influence performance in virtual reality. These are: level of experience, spatial abilities, and human sensory and physiology, which can highly influence several concepts related to task performance, e.g. learnability and effectiveness. Third, one of the main strengths of VR is the possibility of multi-modal interaction and that is the one which has the most difficult technical issues. Fourth, Stanney emphasises the need for new design metaphors in VR, and thinks that it is pointless that we have new technology and devices with old metaphors. Finally, the author highlights that VR usage can cause health and safety problems, like cybersickness, which can influence the usability of VR. "Cybersickness (CS) is a form of motion sickness that occurs as a result of exposure to VEs."

We chose Stanney's factors to be a framework for our research because we think with the help of these factors we can cover the complexity of usability problems in our collaborative virtual environment.

\section{Method}

\subsection{Study Design}

To investigate collaborative information seeking in a heterogeneous virtual environment, we designed a scenario that tasked the two participants in each session to work together to arrange a fictional two-day tour for American students visiting Budapest, Hungary. They were given the following instructions:

"Create a holiday plan for two days for an American student group. Make sure to include in your plan a trip to a bath and a ruin pub. Also, both days should end at a club. Suggest places for lunch and dinner for both days; they receive breakfast at their hotel. Try to fill both days with classic tourist attractions. Create a plan that is varied, yet manageable. Display the final plan in the table located on the front wall." 
The task and the environment were analogous to a real-life situation in which travelers plan a trip from a tourist office. The environment contained posters on the walls promoting local tourist sites, restaurants and bars, a map to help participants identify the location of these objects, a jointly-editable timetable to plan the trip, digital post-it notes for working notes, and decorative items (e.g., tables, window, plants). The task was considered complete when the schedule for both days was finalized.

One of the two participants was in an immersive virtual reality CAVE while the other participant navigated the same space on a 2D display on a desktop computer. (Fig. 1) Both participants worked in the same virtual room with posters, a map, a jointly editable schedule document, and decorative objects, as described above. (Fig. 2) Both participants had access to the same functionality (navigating around the space, moving objects, typing in the editable schedule, highlighting objects, and writing on sticky notes). However, the functions were mapped to different hardware input devices, as afforded by the different environments.

Immediately following completion of the task, participants were interviewed about their experiences with virtual reality more generally and with collaboration in a virtual environment. They were also asked to complete a mental rotation exercise, a demographic and gaming/virtual reality experience questionnaire, an assessment of the collaboration quality and the usability of the space, and their familiarity with Budapest and the locations represented in the posters.

Participants were represented by avatars consisting of a head and an arm. The head included a face helping to indicate which way a participant was facing. The face served as a way for participants to infer where someone was looking and what they were seeing from their perspective. One participant accessed the virtual environment while fully immersed in a CAVE, while the other accessed it from a desktop. Participants were randomly assigned to each mode of interaction. Participants could communicate with one another via headsets connected to Skype. Participants did not know one another prior to the experiment. They were not given a time limit to complete the task; each session, therefore, lasted anywhere from 15 to 50 minutes.

Prior to beginning the task, participants were jointly trained on how to use the system. This training served not only to teach them how to use the equipment and to familiarize them with the user interface, but also to establish a relationship with their partner. They were asked to introduce themselves to their partner and shake their virtual hand. Thus, the process of building common ground started the moment they introduced themselves. Following this, they were instructed to take a poster off the wall and move it to another location in the environment that they believed their partner could see. Next, they were asked to put a note next to the poster they had moved and to write something on it. At the end of each activity, the collaborative partner was asked to provide feedback. Once all activities were 
completed, the participant was asked to return the environment to its default setting. The researcher then read the participants the instructions, outlined above.
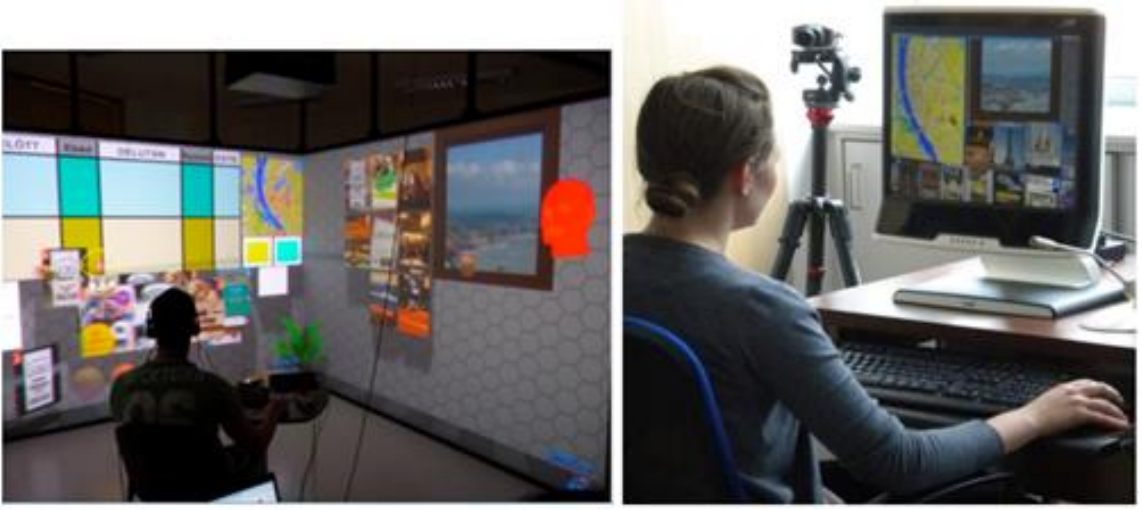

Figure 1

The virtual environment: CAVE (left), Desktop (right)

\subsection{Environment}

As VR software engine, we applied the Virtual Collaboration Arena (VirCA) [8].

Both participants worked in the above mentioned $3 \times 3 \mathrm{~m}$ virtual room, displayed in two fundamentally different ways (Fig. 1).

Participants in the CAVE worked in a $3 \times 3 \mathrm{~m}$ real area surrounded by three projected walls creating a highly immersive experience. They wore INFITEC (Wavelength Multiplex Technology 3D) glasses to see the stereoscopic view, and a headphone with a microphone to communicate with their partner through Skype. On the glasses, a head tracker was also mounted. Depending on the research phase, a wireless, palm sized keyboard and trackpad controller or traditional keyboard and mouse were used to navigate the space and interact with the virtual objects (Table 1). When typing, CAVE participants had to first enter the editing surface with the controller and then use the small mobile keyboard to type. CAVE participants could walk around in the early stage of the research and were seated in later stages.

The desktop participants were seated at a personal computer equipped with a Tobii eyetracking monitor. They viewed the $3 \mathrm{D}$ space on the $2 \mathrm{D}$ monitor in a fashion similar to viewing a virtual world like Second Life and navigated through the space using the regular keyboard and the mouse. They wore a headphone with a microphone to communicate with their partner through Skype. Both participants were represented by an avatar (one blue, one red), consisting of a head and an arm. 
Table 1

The description of each research phase

\begin{tabular}{|c|c|c|c|}
\hline Phase & $\begin{array}{c}\text { No. of } \\
\text { Participants }\end{array}$ & CAVE Environment & Desktop Environment \\
\hline 1 & 40 (20 pairs) & $\begin{array}{c}\text {-participants could walk in the } \\
\text { environment } \\
\text {-manipulation: wireless, palm } \\
\text { sized keyboard and trackpad } \\
\text { controller }\end{array}$ & $\begin{array}{c}\text {-participants were seated in } \\
\text { front of a desktop computer } \\
\text {-manipulation: keyboard } \\
\text { and mouse }\end{array}$ \\
\hline 2 & 42 (21 pairs $)$ & $\begin{array}{c}\text {-participants were seated } \\
\text {-manipulation: keyboard, } \\
\text { mouse }\end{array}$ & \\
\hline 3 & 40 (20 pairs) & \begin{tabular}{c} 
mann \\
\hline
\end{tabular}
\end{tabular}

\subsection{Participants and Phases of the Research}

Participants consisted of 61 pairs of Hungarian university students (58\% male). They ranged in age from 18 to 30 , with a median age of 22.58 years. They were recruited by email and given a 3000 HUF mall voucher (approximately 11 USD) for their participation. In later stages, compensation was increased to account for the increased duration of the experiment.

The 61 pairs of participants took part in three phases of the study. The three phases are listed below with a summary of the phases and demonstrated in Table 1. While there were small changes between the phases to improve the design of the space, the task, and the research goals stayed the same.

\subsection{Analysis}

Data for this paper are from the post-experiment interviews. Interviews were recorded and transcribed in Hungarian. The coding system was developed and refined though several iterative coding phases. We built on Stanney's framework [30] when developing the framework. First, in several group discussions our research group defined the codes and the unit of analysis: utterance. Second, two Hungarian native speakers among the researchers coded the interviews. They used the Atlas.ti.6 software to perform content-analysis. The coding scheme was then refined in a group discussion based on the two coder's experience. This resulted in the identification of 12 codes, a code for each identified usability issue. Third, one native Hungarian speaker analyzed the interviews based on the final coding scheme. During a fourth and final coding pass these were divided into three board categories: (1) VR environment, (2) Device interaction, and (3) Task specific. We then selectively translated representative quotes to English for this paper. 


\section{Results}

In this section we are going to introduce a framework of usability goals based on an evaluation of our collaborative virtual environment. We identified twelve usability themes and we grouped them into three categories based on the underlying context of the usability problem: VR environment, device interaction and task specific problems (Table 2). For each usability theme we describe the theme and why it is important for CVEs and then present examples from the usability study of our system. We used Stanney's framework [30] to develop our initial coding system and we expanded this to include usability goals related to collaboration.

Table 2

Usability themes grouped by the underlying context

\begin{tabular}{|c|c|c|}
\hline VR Environment & Device interaction & Task-specific \\
\hline Visibility & Device usability & Findability \\
\hline Depth perception & Learnability & Informativeness \\
\hline Consistency of views & & Usefulness \\
\hline Presence & & \\
\hline Copresence & & \\
\hline Physical metaphor & & \\
\hline Simulator sickness & & \\
\hline
\end{tabular}

\subsection{VR Environment}

\subsubsection{Visibility}

Visibility refers to the physical visibility of the virtual environment and various elements of the space. Problems related to the users' ability to see the virtual environment and the quality of the images can ruin the user experience, as it takes extra effort from the user to use the system [7]. We collected responses regarding visibility through the interviews. Analysis of video recordings of the interactions can also help identify visibility problems. As Sas and O'Hare [24] stated the higher the graphical resolution, the more realistic the image and thus the more successful the virtual reality space, because of the higher presence feeling of the participants. That is why it is important to explore and examine these problems.

Most of the visibility problems in this space were related to the resolution and the fact that the participants could not read or make out details of text and images because of the low resolution of the displays. Resolution issues emerged both with the posters and the editable document.

It would be great if I could read the posters without zooming. (Phase 3/ Desktop) 
The 3D glasses also interfered with seeing the physical keyboard the participants used to type. This exemplifies the problems of working with physical interaction hardware when wearing 3D glasses or a head-mounted display.

I couldn't see the keyboard because of the 3D glasses. (Phase 1/Cave)

\subsubsection{Depth Perception and Depth Control}

This theme describes participants' problems with the perception of depth in the VR environment and with controlling the movement of objects. In the interviews they reported that the main cause of the problems was the lack of smooth scrolling: the mapping between the mouse scrollwheel movement and the movement in the virtual space was unnatural. A small movement on the mouse scrollwheel resulted in a long and sudden jump in the virtual space.

I had to scroll with the mouse to control the arm, but the scrolling was not smooth. (Phase 1/Cave)

Sometimes it was difficult to grab the posters. My arm reached through them, and I had to scroll back. (Phase 2/Desktop)

The consequence of impaired depth perception was that participants found it difficult to grab posters and put posters into the timetable

\subsubsection{Consistency of Views (Awareness)}

In collaborative systems the consistency of view between two participants is very important in supporting collaboration. Consistency of views refers to perceiving what the other person is looking at. Participants wanted to know where the other person was looking and this joint visual attention was necessary for the collaboration to work smoothly. This was facilitated by the limited avatar of the other person: the head and the arm. Looking at where the head and the arm were pointing helped participants know where their collaborators were looking. When this was not sufficient or not working properly, participants supplemented with verbal descriptions.

I saw that virtual hand, and I could conclude what my partner was doing from the position of it. (Phase 1/ Cave)

I could see his/her hand and head, and s/he also told me what s/he was doing. (Phase 1/Desktop)

However, for some of our participants this was not enough. Several participants mentioned that seeing the head and the hand was not enough, gaze and other nonverbal cues would have helped a lot.

It was difficult... It was disturbing, that we gave little feedback on our partner's actions, and I couldn't see his/her eyes...(Phase 1/ Cave) 
To address this problem, in some cases, one of the partners verbally described where s/he was looking.

\subsubsection{Presence}

Presence is a very important usability goal for virtual environments. During the interview we asked participants to rate how much they felt that they were in the virtual environment on a scale of 0 to 100 .

Our previous results show that participants felt more present in the Cave setting than in the Desktop setting in all three phases.

Table 3

Participants' mean score of presence feeling in each phases

\begin{tabular}{|c|c|c|c|}
\hline & Phase 1 & Phase 2 & Phase 3 \\
\hline $\begin{array}{c}\text { Cave mean score } \\
\text { (standard deviation) }\end{array}$ & 76.6 & 72.12 & 70.32 \\
\hline Desktop mean score & $(22.56)$ & $(17.49)$ & $(22.93)$ \\
(standard deviation) & 59.2 & 63.95 & 61.31 \\
\hline
\end{tabular}

Two main factors seem to have influenced the feeling of presence in our space. First, four participants commented on the quality of the virtual environment: the resolution of the graphics and the usability of the interaction. Second, four participants mentioned that engagement with the task influenced their feeling of presence. These factors reflect Sas and O'Hare's [24] original classification of factors.

The resolution of the 3D VR environment was not good enough (lifelike) for participants and for some of them the control of the system was difficult and decreased the feeling of being present.

I would say 75, because the graphics of the room was not as realistic as it would be possible... (Phase 3/Cave)

Participants also reported that the more they were engaged with the task, the more present they felt in the space. As the next participant described this:

I would say 60-70, because it is a desktop, but I felt present. I think it is like gaming, if you really concentrate on the game, you'll feel like you are being there. (Phase 1/ Desktop)

There was another factor that disturbed the feeling of presence: participants reported that sometimes they were too aware of being in an artificial VR cave, so they couldn't feel present.

I would say 70, but I was aware of that it was not a real environment. (Phase 1/Cave) 


\subsubsection{Copresence}

The feeling of co-presence is the participants' subjective feeling of the other participant was there/ awareness. In the interview participants were asked to report their feeling of co-presences on a scale of 0 to 100 (Table 4). Participants felt their partner more copresent in the Desktop setting than in the Cave setting in all three phases. This result is the opposite of the presence result. We think that this is because the participants correlate their experience (feeling of copresence of the partner) to their presence experience. As Cave participants felt more present, correlated to these high values they felt their partner less present. This is also the case for Desktop participants, just in the opposite way.

Participants reported that the following two factors helped the feeling of copresence the most: hearing the other participant's voice and seeing his/her avatar's hand. They also reported that collaboration itself made them feel that the other participant was there.

Table 4

Participants' mean score of copresence feeling in each phases

\begin{tabular}{|c|c|c|c|}
\hline & Phase 1 & Phase 2 & Phase 3 \\
\hline $\begin{array}{c}\text { Cave mean score } \\
\text { (standard deviation) }\end{array}$ & 54.6 & 65.8 & 69.2 \\
\hline $\begin{array}{c}\text { Desktop mean score } \\
\text { (standard deviation) }\end{array}$ & $(28.13)$ & $(22.56)$ & $(25.01)$ \\
\hline
\end{tabular}

I saw the other participant, and it might sound stupid, but I felt that s/he was there from how s/he talked and what s/he said. (Phase 1/Desktop)

But in some cases these two things were not enough, and participants didn't feel the copresence of the other.

Sometimes I just saw that a head or a hand go before me. (Phase 1/Cave)

\subsubsection{Physical Metaphor}

Metaphors are "understanding and experiencing one kind of thing in terms of another" (Lakoff and Johnson in [10]). The most important role of metaphors in HCI (Human Computer Interaction) is "to transfer knowledge from a source domain (familiar area) to a target domain (unfamiliar area) [10].

In his article Stanney [30] states that in virtual reality new metaphors are needed because metaphors transferred from older forms of technology can have strong limitations.

Participants reported several issues which confused them because of their expectations of how the VR space would work based on the physical metaphor of 
a room - that in the VR environment the same rules would be valid as in the real world. They reported these in the interviews.

First in phase 1, it was unusual to "write on the wall" (write in an editable document on the wall).

It is really strange to write on the wall into a word document. (Phase 1/ Cave)

Second, it was possible to reach through things in the VR environment. It was possible to reach through a poster (and as a consequence difficult to grab it) and reach through the wall (as a consequence put a poster behind the wall). Besides, it was also possible to go through the partner's avatar, which was "creepy" according to the participants' opinions.

It was a bit difficult that I could put the poster behind the wall, I mean the system didn't put it automatically on the wall. Instead I could reach through the wall. (Phase 3/Desktop)

In addition, participants reported that posters didn't fit exactly in the schedule table and they could hover in the VR environment, which was also surprising.

I might place the posters in the schedule in a wrong way, or the posters didn't fit perfectly in the schedule. (Phase 2/Cave)

While using a familiar physical metaphor can help users interact with new technologies, metaphors can also confuse users when the technology breaks the rules of the physical metaphor. Choosing a metaphor carefully and evaluating it to understand how users interpret it is crucial when designing VR environments.

\subsubsection{Simulator Sickness}

Simulator sickness is an important aspect of virtual reality usability as it can not only ruin the user experience but make the use of a VR environment impossible for some users. Fortunately, in our experiment participants reported simulator sickness in only a few cases. Most utterances (18/22) are from the Cave participants' interviews. The typical symptoms were just mild discomfort, like the tiredness of the eyes, pain in the eyes, dizziness and headache (after taking off the 3D glasses).

My eyes are tired, no dizziness, it was just a bit tiring for my eyes. (Phase 2/Cave)

It is important that simulator sickness can become a serious problem for users [18]. So designers should consider these results when designing a virtual reality environment for different users. 


\subsection{Usability Dimensions Related to Device Interaction}

\subsubsection{Device Usability}

Finding the appropriate interactive input and output devices and mappings for interaction with VR environments is a difficult challenge for both navigating through the space and interacting with objects. The user moves through the space via interacting with an input device instead of physically moving their body and grabs and manipulates objects through the same or a different input device. To find the appropriate device and interaction method for these complex actions is a challenge for design.

Several usability problems occurred related to different input devices, in most cases the keyboard and the mouse, but there were also some issues related to the 3D glasses in our environment. In phase 1 the Cave participants reported that the keyboard and the keyboard buttons were too small, and it was difficult to type as a result of it. In addition, the keyboard was not sensitive enough.

Typing, and using the small keys of the keyboard of this device was difficult. Sometimes it was not sensitive enough, which was also a problem. (Phase /Cave)

In addition, for Desktop participants it was difficult to control the system with a mouse.

After FPS* (*First Person Shooting) ...it was strange to get used to the coordination (with the mouse). (Phase 2/Desktop)

In phases $2 \& 3$ it was difficult to see the keyboard because the $3 \mathrm{D}$ glasses covered a lot from the field of view.

I couldn't see the keyboard because of the 3D glasses. (Phase 1/Cave)

\subsubsection{Learnability and Memorability}

Learnability and memorability are central concepts of usability and are closely linked to how natural a system is to use. Learnability describes how easily and quickly users can learn to use a system while memorability describes how well users can recall this knowledge later. As described in the previous sections, understanding and operating VR environments is challenging and the more natural the interactions can be, the faster users can learn it and the longer they will remember it. Learnability of the system control has a key role because before a participant can fully use a system and collaborate with the partner, s/he needs to learn how to use it. In our study participants were allowed a time period to learn to use the system.

The majority of our participants agreed that while interaction was first awkward and took some time to learn, after some practice the interaction became more 
natural. As VR environments are complex systems in terms of interaction, users should always be given an opportunity to learn and practice on the system.

At the beginning coordination was difficult, then it became easier. It is like driving a car, first you have to think before every action, then it becomes a routine. (Phase 1/ Cave)

\subsection{Task Specific Usability Factors}

The usability goals described so far can be applied to most CVEs. This section, however, describes usability goals that are specific to the user tasks each CVE supports. With the exception of usefulness and utility, the usability goals of our environment were related to supporting the information seeking and sharing goals.

\subsubsection{Findability}

The goal of our system was to provide textual and graphical information to users to solve information problems. Thus the layout of this information in the space was an important factor. The posters were laid out on the left wall in topical groups, the middle wall included the schedule and the map, as shown in Figure 2.

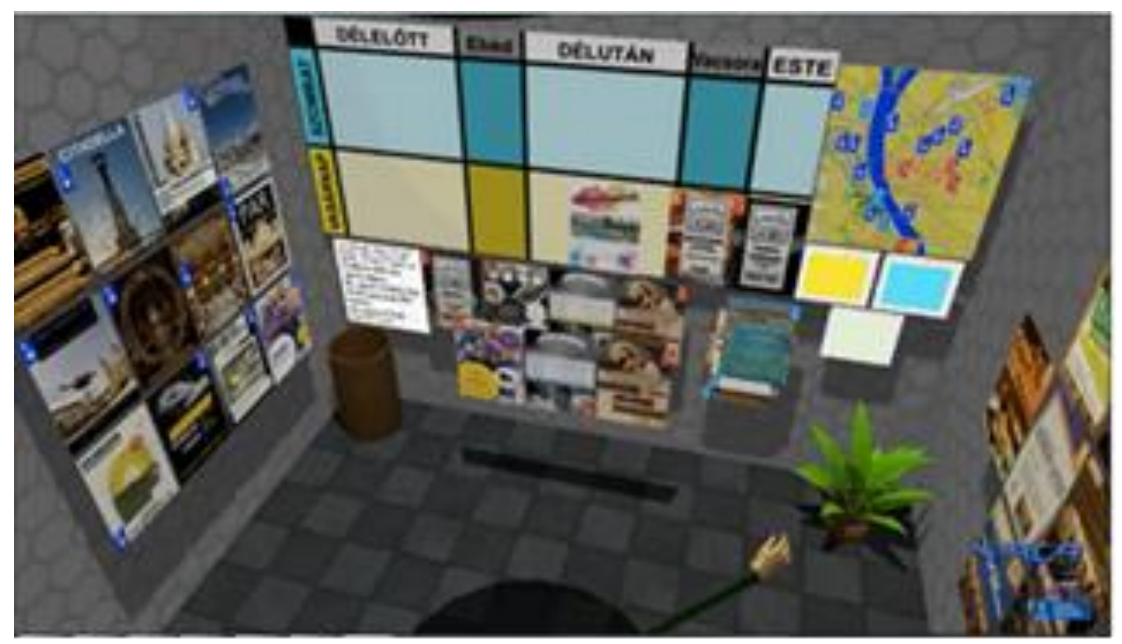

Figure 2

The layout of information in the VR environment

Finding information to solve a task was essential for participants to be successful. Thus findability of information was an important task-related usability factor for our study. In our experiment participants mentioned two main factors that influenced the findability of information in the virtual environment: the position of the posters and the icons on the posters. 
The position of the posters divided the participants' opinions: some of them said, that the themed position of the posters helped - it means that posters of the same category, e.g. restaurants, party places, sightseeing events were grouped together on the walls of the VR room.

I think this system is transparent...The posters position in the environment is good, they are easy to find.( Phase 2/Desktop)

In contrast, some participants found the environment crowded, and told us that it was difficult to find the posters and keep everything in mind. In addition, they also found it difficult to handle three walls of information at the same time.

It was difficult to watch three walls at once, and search for things. (Phase 3/ Desktop)

Furthermore, participants mentioned that the icons on the posters and the map helped them a lot. There were 4 kinds of icons on the posters: star for sightseeing possibilities, fork\&knife for restaurants, wave for baths, note for music/party places. But, some participants found it difficult to interpret the icons on the map and to find the location of the posters on the map with the help of the icons.

I don't know why, it might be the colour, or the icons, but it was difficult for me to interpret the map. (Phase 1/Cave)

I think the meaning of the icons on the map were clear. (Phase 2/ Cave)

In future research it will be interesting to examine what factors influence users' preferences for the layout of information in 3D virtual spaces.

\subsubsection{Informativeness}

Another task-related usability goal in our study was informativeness, or the right amount of information that leads to success. According to our participants' feedback, the posters and the map were informative enough to solve the task. The icons on the posters and the map helped a lot, but sometimes their meaning were not clear (3 no5 posters with different colours).

I loved the icons. The star meant sightseeing and I think posters with fork\&spoon icon were restaurants... (Phase 1/Cave)

Participants said that more information would be helpful on some posters, e.g. the price of the tickets, what to expect in a place (music, food).

Some of the posters didn't tell much information about what to expect at that place. (Phase 3/ Cave)

As with the layout of the information, the amount and access to the information can be further examined in future research. 


\subsubsection{Usefulness}

While the layout and the amount of information above address the usability of the information in the space, this factor discusses the usefulness of features, whether the features included in the system serve user goals. While the terminology for describing this concept in the usability literature is somewhat mixed, we will use Nielsen's [20] definition of utility: "whether it provides the features you need" or not. In their book Rubin and Chisnell [23] refer to the same concept using different terms and define usability and usefulness as: Usability is: "when a product or service is truly usable, the user can do what he or she wants to do the way he or she expects to be able to do it, without hindrance, hesitation, or questions." ([23] p. 4). "Usefulness concerns the degree to which a product enables a user to achieve his or her goals, and is an assessment of the user's willingness to use the product at all ([23] p. 4)

The utility/usefulness factor refers to the value judgment of a function (according to the participants if it is useful or not). 276 utterances belong to this factor, $67 \%$ of them are negative. Negative utterances are functions which participants didn't find useful during task-solving. In this case, the functions are the items of the virtual environment which were designed to help the participants to solve the task. While we collected this data through interviews, we also asked participants to evaluate the utility of these features through a survey instrument. In our study the following items were evaluated: the map, the posters, the highlight function, and the notes.

$72 \%$ of the participants found the map useful, except those who had a broad knowledge of Budapest. The same is true for the posters, they were useful, but sometimes $(32 \%)$ just for giving ideas. $88 \%$ of the participants did not find the highlight function useful, because it was not natural and took extra time to use, so it is not surprising that sometimes they even forgot about it. Besides, they knew what aspect of the virtual reality they were talking about because they continuously communicated verbally via Skype. In some cases, they just drew the attention of their partner to something just by pointing at it or putting it in the middle of the room. $87 \%$ of the participants did not like to use the notes, either, except for adding extra events. They said the task was not that difficult, they didn't have to remember things and it didn't take that long that it would be useful to use on notes. Besides, as written above, participants communicated via Skype, so the notes were not useful for communication, either.

The map and the posters are easy to use... I liked them because they were colourful and raise awareness (Phase 2/Desktop)

We could perfectly solve the task by talking...we didn't use highlight, instead we put things forward (in the field of view of the partner) (Phase 2/Cave)

Because talking was faster than typing (Phase 1/Desktop) 
Examining the utility of the task-related elements of the environment is crucial in creating a system that will help achieve users' goals. These factors will be different for each VR environment but should be included among the design goals and usability evaluation of the system.

\section{Discussion \& Conclusions}

In this paper we presented a framework of usability goals for collaborative virtual environments based on a review of the literature and a usability study of a CVE. We used Stanney's [30] framework as a starting point and we transformed it based on our qualitative data from user interviews. We identified twelve usability goals grouped in three main categories, based on the previous literature about collaborative virtual environments', Stanney's framework and our results.

Stanney defines five main issues one has to have in mind when designing a virtual reality environment: task characteristics, user characteristics, multi-modal interaction, new design metaphors and health and safety issues. Stanney stated that these issues are important in all kinds of virtual environments, so this framework is highly generalizable. In our paper we refined and expanded Stanney's framework to be able to cover all important design aspects of collaborative virtual environments: our category of "Device interaction" expands Stanney's category of "Multimodal interaction", and "VR environment" expands "New design metaphors". Previous research about usability problems in collaborative virtual environments also confirm our results. Usability problems in the areas of learnability [12] [32] visibility [11] [13] [32] awareness [31] [13] [32] device usability [12] and simulator sickness [19] were typical usability problems in several collaborative virtual reality environments. However, no comprehensive usability framework was described in these studies.

Collaborative virtual environments (CVE) pose special challenges for designers. In addition to accommodating user needs for single-user virtual environments, CVEs require tools to support awareness of others' actions, the feeling of copresence, and collaboration. This paper presented an expanded usability framework for CVEs based on a review of the literature and a usability study of one specific CVE. In future research we will continue the formalization of the framework, we will integrate usability goals from other collaborative systems, and assess the usefulness of our framework in the design and evaluation of CVEs. For our future experiments, we plan to use the successor of the VirCA system applied now: the MaxWhere VR platform [3] [5] [6] [14] [16] promises new prospects.

\section{Acknowledgement}

This study is supported by the KTIA_AIK_12-1-2013-0037 project: Virtual NeuroCognitive Space for research and development of future immersive mediatechnologies (NeuroCogSpace). The project is supported by Hungarian Government, managed by the National Development Agency/Ministry, and financed by the Research and Technology Innovation Fund. 


\section{References}

[1] Baranyi, P., \& Csapo, A. (2012) Definition and Synergies of Cognitive Infocommunications. Acta Polytechnica Hungarica, 9(1), 67-83

[2] Baranyi, P., Csapo, A., \& Varlaki, P. (2014) An Overview of Research Trends in Coginfocom. In Intelligent Engineering Systems (INES) 2014 $18^{\text {th }}$ International Conference on (pp. 181-186) IEEE

[3] Biró, K., Molnár, Gy., Pap, D., Szüts, Z. (2017) The Effects of Virtual and Augmented Learning Environments on the Learning Process in Secondary School, $8^{\text {th }}$ IEEE International Conference on Cognitive Infocommunications, Debrecen, 2017 (pp. 371-376) IEEE

[4] Bowman D. A., Kruijff E., LaViola J. J. \& Poupyrev I. (2004) 3D User Interfaces: Theory and Practice. Redwood City, CA: Addison Wesley Longman Publishing Co.

[5] Budai, T., \& Kuczmann, M. (2018) Towards a Modern, Integrated Virtual Laboratory System. Acta Polytechnica Hungarica, 15(3), 191-204

[6] Bujdosó, Gy., Novac, O. C., \& Szimkovics, T. (2017) Developing Cognitive Processes for Improving Inventive Thinking in System Development using a Collaborative Virtual Reality System, $8^{\text {th }}$ IEEE International Conference on Cognitive Infocommunications, Debrecen, 2017 (pp. 79-84) IEEE

[7] Cruz-Neira, C., Sandin, D. J., \& DeFanti, T. A. (1993, September) Surround-Screen Projection-based Virtual Reality: the Design and Implementation of the CAVE. In Proceedings of the $20^{\text {th }}$ annual conference on Computer graphics and interactive techniques (pp. 135-142) ACM

[8] Galambos, P., Baranyi, P., \& Rudas, I. J. (2014) Merged Physical and Virtual Reality in Collaborative Virtual Workspaces: The VirCA Approach. In Industrial Electronics Society, IECON 2014, 40 ${ }^{\text {th }}$ Annual Conference of the IEEE (pp. 2585-2590) IEEE

[9] Heeter, C. (2003) Reflections on Real Presence by a Virtual Person. Presence: Teleoperators and Virtual Environments, 12(4), 335-345

[10] Helander, M., Landauer, T., \& Prabhu, P. (1997) Mental Models and User Models. In Handbook of human-computer interaction (pp. 49-63) Elsevier

[11] Heldal I., Schroeder R., Steed A., Axelsson, A., S., Spante, M., \& Wideströ J. (2004) Collaboration and Immersiveness in Shared Virtual Environments: A Comparison in Performance and Interaction in Five Settings. In The usability of collaborative virtual environments (pp. 110122) Saarbrücken, Deutschland

[12] Heldal, I. \& Schroeder, R. (2002) Performance and Collaboration in Virtual Environments for Visualizing Large Complex Models: Comparing Immersive and Desktop Systems. Proceedings of the $8^{\text {th }}$ International 
Conference on Virtual Systems and Multimedia (VSMM 2002) Gyeongju, Korea, September 2002, 208-220

[13] Heldal, I. (2004) Usability Development for Collaborative Virtual Environments. Virtual Reality Design and Evaluation Workshop 2004, (October) 1-8, Retrieved from

http://www.view.iao.fraunhofer.de/Proceedings/papers/heldal.PDF

[14] Horvath, I., \& Sudar, A. (2018) Factors Contributing to the Enhanced Performance of the MaxWhere 3D VR Platform in the Distribution of Digital Information. Acta Polytechnica Hungarica, 15(3) 149-173

[15] Kohonen-Aho, L., \& Alin, P. (2015) Introducing a Video-based Strategy for Theorizing Social Presence Emergence in 3D Virtual Environments. Presence, 24(2) 113-131

[16] Kövecses-Gösi, V. (2018) Cooperative Learning in VR Environment. Acta Polytechnica Hungarica, 15(3), 205-224

[17] Lányi, S. C. (2014) The Thousand Faces of Virtual Reality. Rijeka: InTech

[18] LaViola Jr, J. J. (2000) A Discussion of Cybersickness in Virtual Environments. ACM SIGCHI Bulletin, 32(1), 47-56

[19] Munafo, J., Diedrick, M. \& Stoffregen, T. A. (2017) The Virtual Reality Head-mounted Display Oculus Rift Induces Motion Sickness and is Sexist in Its Effects. Experimental Brain Research, 235 (889)

[20] Nielsen, J. (1994) Usability engineering. Elsevier

[21] Nilsson A., Heldal I., Schroeder R., \& Axelsson A. S. (2001) The LongTerm Uses of Shared Virtual Environments: An Exploratory Study. IN Heldal I. (2010) The usability of collaborative virtual environments: Towards an evaluation framework. Saarbrücken, Germany, Lambert Academic Publishing AG \& Co

[22] O'Leary, D. E. (2008) Gartner's Hype Cycle and Information System Research Issues. International Journal of Accounting Information Systems, $9(4), 240-252$

[23] Rubin, J., \& Chisnell, D. (2008) Handbook of Usability Testing: How to Plan, Design and Conduct Effective Tests. John Wiley \& Sons

[24] Sas, C., \& O'Hare, G. M. (2003) Presence Equation: An Investigation into Cognitive Factors Underlying Presence. Presence: Teleoperators and Virtual Environments, 12(5), 523-537

[25] Schroeder, R. (2002) Copresence and Interaction in Virtual Environments: An Overview of the Range of Issues. Presence 2002: Fifth International Workshop, 274-295. Retrieved from http://users.ox.ac.uk/ inet0032/papers/copresence and interaction 2002.pdf 
[26] Schroeder, R. (2006) Being There Together and the Future of connected presence. Presence: Teleoperators and Virtual Environments, 15(4), 438454

[27] Sheridan, T. B. (1992) Musings on telepresence and virtual presence. Presence: Teleoperators and Virtual Environments, 1(1), 120-126

[28] Shim, W., \& Kim, G. (2003) Designing for Presence and Performance: The Case of the Virtual Fish Tank. Presence: Teleoperators and Virtual Environments, 12(4), 374-387

[29] Slater, M. (2004) How Colorful Was Your Day? Why Questionnaires Cannot Assess Presence in Virtual Environments. Presence Teleoperators and Virtual Environments, 13(4), 484-493

[30] Stanney, K. (1995) Realizing the Full Potential of Virtual Reality: Human Factors\Issues that could Stand in the Way. Proceedings Virtual Reality Annual International Symposium '95

[31] Sutcliffe, A., \& Alrayes, A. (2012) Investigating User Experience in Second Life for collaborative learning. International Journal of Human Computer Studies, 70(7), 508-525

[32] Tromp, J. G., Steed, A., \& Wilson, J. R. (2003) Systematic Usability Evaluation and Design Issues for Collaborative Virtual Environments. Presence: Teleoperators and Virtual Environments, 12(3), 241-267

[33] Virvou, M., \& Katsionis, G. (2008) On the Usability and Likeability of Virtual Reality Games for Education: The Case of VR-ENGAGE, 50, 154178 